\title{
Characterization of SEM speckle pattern marking and imaging distortion by Digital Image Correlation
}

\author{
Adrien Guery ${ }^{1,2}$, Félix Latourte ${ }^{1}$, François Hild ${ }^{2}$ and Stéphane Roux ${ }^{2}$ \\ ${ }^{1}$ EDF R\&D, Site des Renardières, avenue des Renardières - Ecuelles, \\ F-77818 Moret-sur-Loing (FRANCE) \\ 2 LMT-Cachan (ENS Cachan/CNRS/UPMC/PRES UniverSud Paris) \\ 61 avenue du Président Wilson, F-94235 Cachan (FRANCE) \\ E-mail: guery@lmt . ens-cachan.fr, felix. latourte@edf. fr, \\ hild@lmt.ens-cachan.fr, stephane.roux@lmt.ens-cachan.fr
}

\begin{abstract}
Surface patterning by e-beam lithography and SEM imaging distortions are studied via digital image correlation. The global distortions from the reference pattern, which has been numerically generated, are first quantified from a digital image correlation procedure between the (virtual) reference pattern and the actual SEM image both in secondary and backscattered electron imaging modes. These distortions result from both patterning and imaging techniques. These two contributions can be separated (without resorting to an external caliper) based on images of the same patterned surface acquired at different orientations. Patterning distortions are much smaller than those due to imaging on wide field images.
\end{abstract}

Keywords: SEM, distortions, e-beam lithography patterning, Digital Image Correlation 


\section{Introduction}

During the last decades, the mechanical behavior of metals at microstructural scales has been increasingly studied with numerical or experimental approaches [1,2]. With the development of Digital Image Correlation (DIC), kinematic measurements via SEM imaging can be performed to provide spatially dense experimental 2D [3, 4], or even 3D [5, 6] fields. These measurements allow for many possibilities, as for instance the coupling with simulations to identify material parameters [7]. However, SEM imaging induces distortions of different natures (e.g., drift or spatial) of the observed object and noise $[3,4,5,8,9,10]$ that are due to the electromagnetic environment of imaging, and that need to be considered to quantify the errors of DIC measurements [5].

Kinematic measurements with DIC require gray level texture images whose dynamic range should be as large as possible with high local contrast. If the natural texture of the material does not provide such contrast, different marking techniques can produce the suited artificial texture on the surface of interest. In the context of SEM imaging, the deposition of microgrids by microlithography is a viable technique $[11,12]$. However the spatial periodicity of the grid may induce displacement measurement errors or poorer spatial resolutions [13]. Investigations have been conducted on the development of random patterns [14] while speckles for SEM imaging can be obtained by remodeling of metallic films [15, 16, 17], by nanoparticles deposition techniques [18], or even by UV-photolithography [17].

An alternative solution described in this paper is also based on random patterning. The particularity is that the pattern is computer-generated before being deposited onto the specimen surface by microlithography. This procedure allows us to parameterize the desired pattern, and leads to a precise knowledge of the "ideal" pattern (referred to as reference pattern in the following). An evaluation of pattern and imaging distortions is then possible by comparing an SEM image of the pattern and its reference counterpart.

The paper is organized as follows. First, Section 2 is devoted to the presentation of the material and the adopted method of microlithographic gold deposition marking. Then, the reference and observed patterns are registered using DIC in Section 3. SEM distortions are evaluated from a series of rotations of the sample and of the scan beam. Finally, although marking and imaging errors affect each image, collecting several of them after a physical rotation of the specimen in the SEM leads to the possibility of separating marking and imaging errors from the global distortion (Section 4).

\section{Materials and methods}

\subsection{Scanning Electron Microscope (SEM)}

The SEM used in this study is an FEI Quanta FEG 600. For imaging, Secondary Electron (SE) Everhart-Thornley Detector (ETD) and Backscattered Electron (BSE) detector in Zcontrast have been used, both with an acceleration voltage of $10 \mathrm{kV}$ and a working distance of $14 \mathrm{~mm}$. The physical size of one pixel is $240 \mathrm{~nm}$. This resolution was chosen for subsequent mechanical analyses so that a sufficient number of grains could be observed. 


\subsection{Microlithographic gold deposition marking}

Electron beam lithography is classically employed in the production of microelectronic components [19]. The technique is based on the selective removal of the "resist" film where it has been exposed to the e-beam according to a specific pattern. In solid mechanics, this technique has been selected to mark in-situ specimen for kinematic measurements [3, 4, 7, 11, 12]. As for photolithography, two types of resist films can be used to achieve etching. A positive one that becomes soluble (depolymerized) once exposed to the electron beam, or a negative one, which becomes insoluble. For this study, a positive resist has been chosen.

The material of this study is 316LN austenitic stainless steel. The aim is to deposit a gold pattern onto the surface of interest of samples, such as an in-situ tensile test sample to measure displacement fields during loading. A mechanical polishing of this surface is performed beforehand with cloths and diamond solutions down to $1 \mu \mathrm{m}$, and with finish with $50 \mathrm{~nm}$ colloidal silica suspension.

The process of microlithography gold patterning is divided into five steps (Figure 1):

(i) The "resist" film — in the present case, a thin layer of polymethylmethacrylate (PMMA) - is synthesized (cured at $180^{\circ} \mathrm{C}$ for $90 \mathrm{~s}$ ) from a precursor resin spin-coated on the surface.

(ii) A dedicated software of the SEM, Raith Elphy Quantum, controls the stage position, the magnification, the electron beam blanking, and the electron beam deflection to write the generated pattern. The marked domain is chosen to be a $400 \times 400 \mu \mathrm{m}^{2}$ square allowing the exposure to be performed with a fixed stage position, by electron beam deflection from the center of the region of interest. The marking consists of many elementary patterns, e.g., circular disks in the present case. The electron beam is unblanked at the coordinates of each disk center. Details about the pattern used in this study are given in Section 2.3. A beam current of $50 \mathrm{pA}$, an acceleration voltage of $25 \mathrm{kV}$ and a working distance of $10 \mathrm{~mm}$ have been selected.

(iii) The third step is the development of the pattern. The exposed resist is dissolved by immersion in a developer solution for $60 \mathrm{~s}$ followed by $30 \mathrm{~s}$ in isopropanol to stop the development. The bare specimen surface is obtained where the resist film was exposed to the electron beam.

(iv) A $20 \mathrm{~nm}$ thick gold film is then deposited across the entire sample surface, using a cathodic deposition apparatus with $40 \mathrm{~mA}$ current.

(v) Finally, the gold capped residual resist is dissolved with a remover solution, leaving the gold film only where it is in direct contact with the substrate. Ultrasound-enhanced cleaning steps are repeated several times. The result is a gold pattern marked in the region of interest for SEM imaging (Figure 2). 


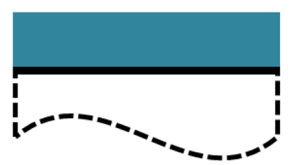

(a)

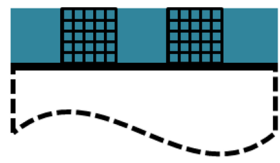

(b)

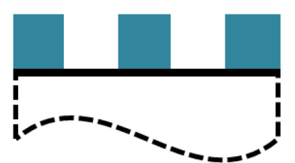

(c)

Resist

Exposed resist Deposited metal

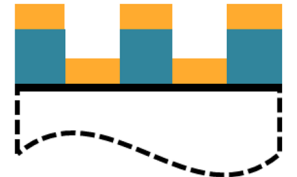

(d)

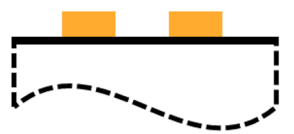

(e)

Figure 1. The five major steps of microlithographic gold patterning. Resin film deposition (a), electron beam exposure (b), development (c), gold deposition (d), dissolution of residual resist film (e)

\subsection{Parameterized pattern}

The software used to control the SEM beam exposure offers a wide range of possibilities for the pattern design. The choice made in this paper is to mark the surface with a known distribution of disk-shaped gold spots of random radius and position. The variation of radius is achieved via modulation of exposure time to electron beam. The number of spots is adjusted to get suitable density of surface covered by gold in the region of interest - in the present case, $17 \%$ (Figure 2(c)) - aiming for an appropriate gray level histogram of SEM images suitable for DIC. A specific visual marking is set in three corners of the region covered by the speckle to get easily the orientation of the marked pattern during observation.

One advantage of using a known pattern in this technique of microlithographic gold deposition marking is that the SEM image of the speckle can be compared with the ideal reference to measure the errors resulting both from lithography and image acquisition with the SEM.

\section{Quantification of systematic error in marking and imaging}

\subsection{Comparison between reference and observed pattern}

The deposited gold speckle is observed with the SEM, using SE (Figure 2(a)) or BSE (Figure 2(b)) detectors. The binary reference that was used to create the speckle pattern is shown in Figure 2(c).

An ideal image of the speckle, designated as the "reference," is generated from the known distribution of spots (Figure 2(c)). Each spot is assumed to have a Gaussian-shaped distribution of gray levels. A typical histogram of SEM images of the speckle shows two peaks (Figure 3). The first peak corresponds to the mean gray level of the uncovered steel surface, and the second one to the mean gray level of the gold disks. These identified gray levels are used to generate the reference (synthetic) image of uniform background and Gaussianshaped spots, as shown in Figure 4(a) for the SE image. For BSE images of the speckle, the underlying microstructure (twins in the present case) appears through the variation of 


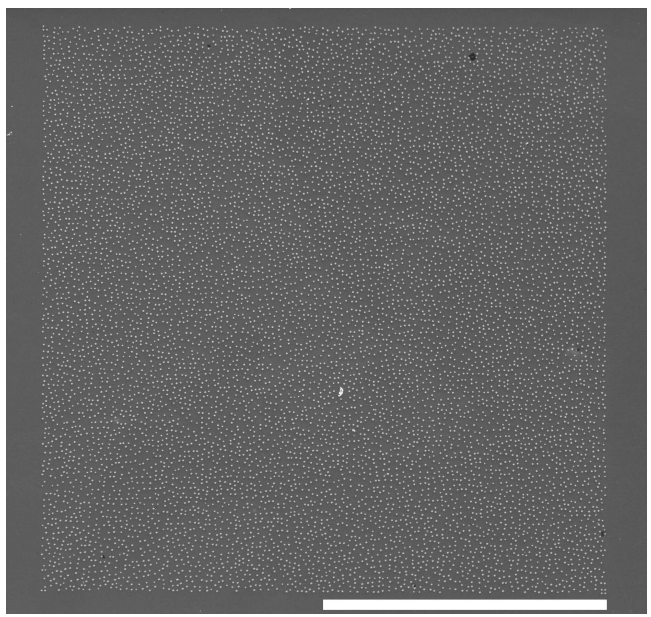

(a)

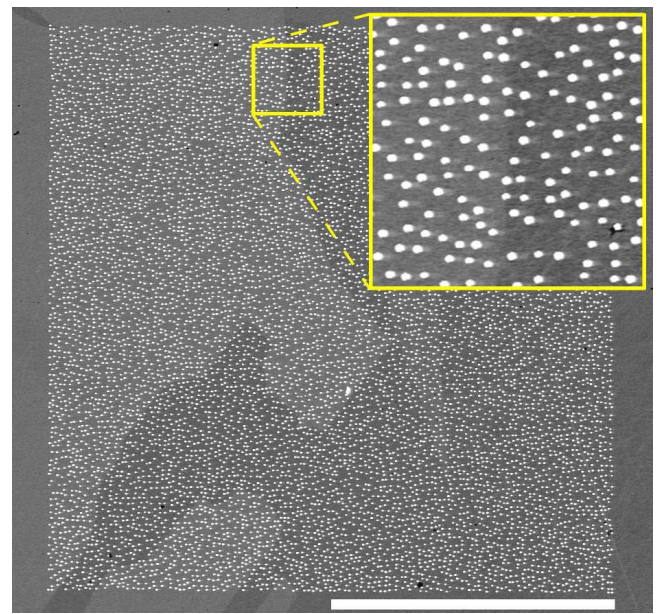

(b)

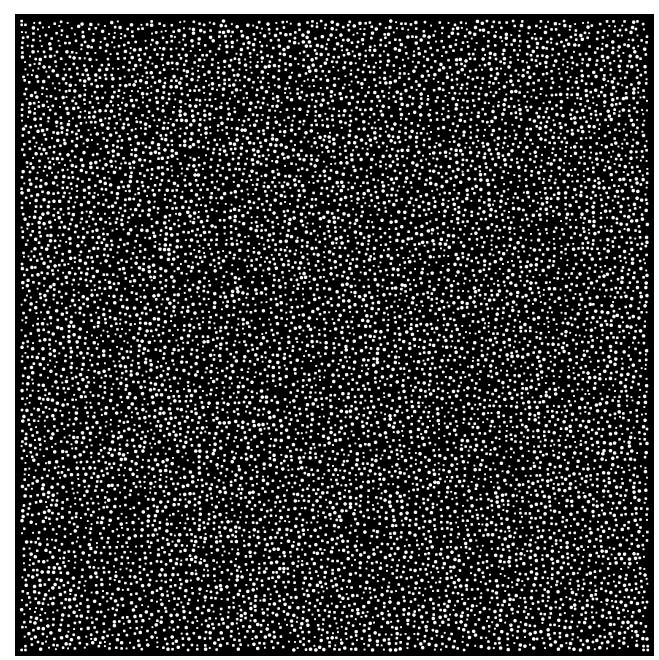

(c)

Figure 2. SEM imaging of the gold speckle with SE (a) and BSE (b) detectors. The scale bar is $200 \mu \mathrm{m}$. Binary image of the reference pattern (c)

the gray levels. The corresponding ideal image is therefore built regionally, namely, each element, twin or grain, of the appearing microstructure is processed with the same method as previously. The result is shown in Figure 4(b).

The displacement fields are measured between an SEM image and the corresponding reference image of the speckle with finite element based DIC [20, 21]. It is convenient for the following developments to resort to a mapping of the image coordinate system $(x, y)$ to the complex plane $z=(x+i y) / L$, where $L$ is the window size. The formulation is based on the texture conservation written with respect to the reference (complex-valued) coordinate systems, $\boldsymbol{z}$. In the transformation, a point $\boldsymbol{z}$ of the reference image $f$ moves to position $\boldsymbol{Z}(\boldsymbol{z})$ in the SEM image $g$

$$
g(\boldsymbol{Z}(\boldsymbol{z}))=f(\boldsymbol{z})
$$




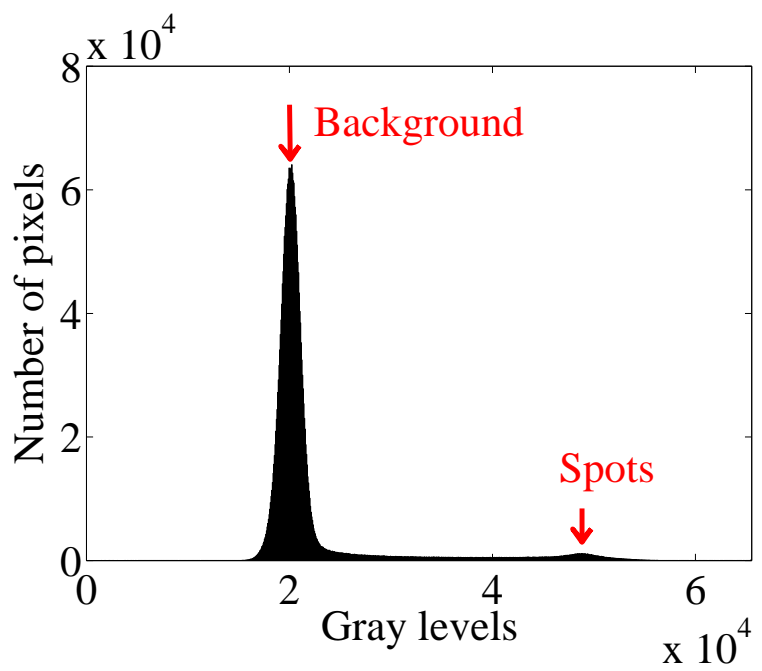

Figure 3. Gray levels histogram of the SE image of the marked region

where the Lagrangian displacement $\boldsymbol{u}$ (here again mapped into the complex plane) is defined by

$$
Z(z)=z+u(z)
$$

The minimization of the sum of squared differences $\rho_{c}^{2}=\left(f(z)-g(z+\boldsymbol{u}(\boldsymbol{z}))^{2}\right.$ of gray levels of the two images is performed by successive (indexed by $n$ ) solutions to the linear system in the incremental displacement vector $\left\{\boldsymbol{\delta} \boldsymbol{u}^{(n)}\right\}$ [22]

$$
[\boldsymbol{M}]\left\{\boldsymbol{\delta} \boldsymbol{u}^{(n)}\right\}=\left\{\boldsymbol{b}^{(n)}\right\}
$$

where $\{\boldsymbol{\delta} \boldsymbol{u}\}$ is the column vector gathering all the unknown amplitudes $u_{k}$ when the displacement field, $\boldsymbol{u}$, is written as

$$
\boldsymbol{u}(\boldsymbol{z})=\sum_{k} u_{k} \psi_{k}(\boldsymbol{z})
$$

where $\psi_{k}(\boldsymbol{z})$ denote the chosen trial displacement fields.

The matrix $[\boldsymbol{M}]$ and the vector $\left\{\boldsymbol{b}^{(n)}\right\}$ are known quantities, calculated over the whole region of interest (ROI), from the gray levels $f$ and $g$ of respectively the reference image and the deformed image, such that

$$
\begin{aligned}
& M_{k l}=\sum_{R O I}\left(\psi_{k} \cdot \nabla f\right)(z)\left(\psi_{l} \cdot \nabla f\right)(z) \\
& b_{k}=\sum_{R O I}\left(f-\tilde{g}^{(n)}\right)(z)\left(\boldsymbol{\psi}_{k} \cdot \nabla f\right)(z)
\end{aligned}
$$

where $\tilde{g}^{(n)}$ is the deformed image corrected by the current estimation of the displacement field $\left(\tilde{g}^{(n)}(\boldsymbol{z})=g\left(\boldsymbol{z}+\boldsymbol{u}^{(n)}(\boldsymbol{z})\right)\right)$. The displacement discretization is performed using an unstructured mesh (Figure 5), built with 3-noded triangular elements (about 40 pixel / $9.6 \mu \mathrm{m}$ long sides). In the following, each DIC calculation will be conducted using this mesh. The adopted 


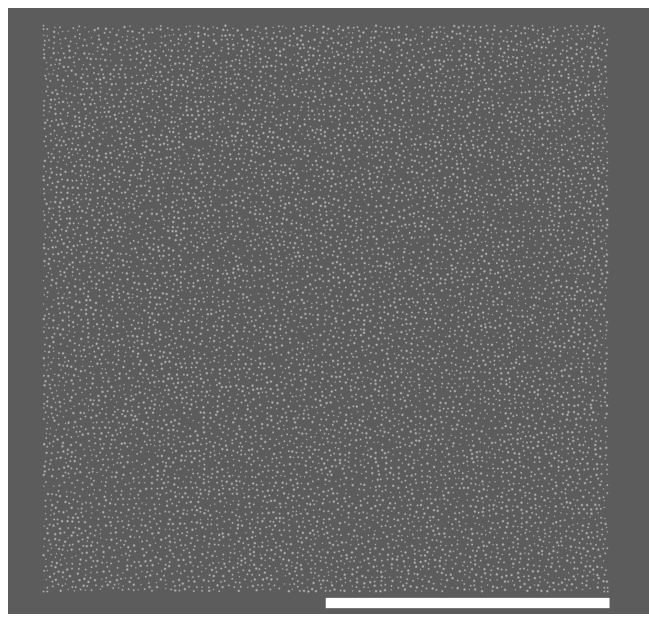

(a)

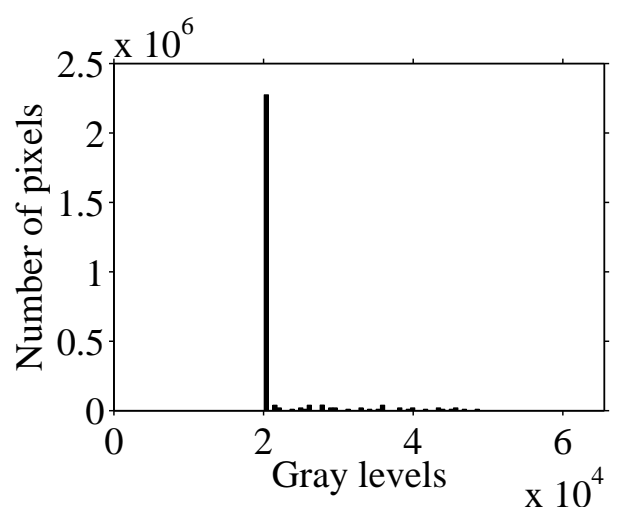

(c)

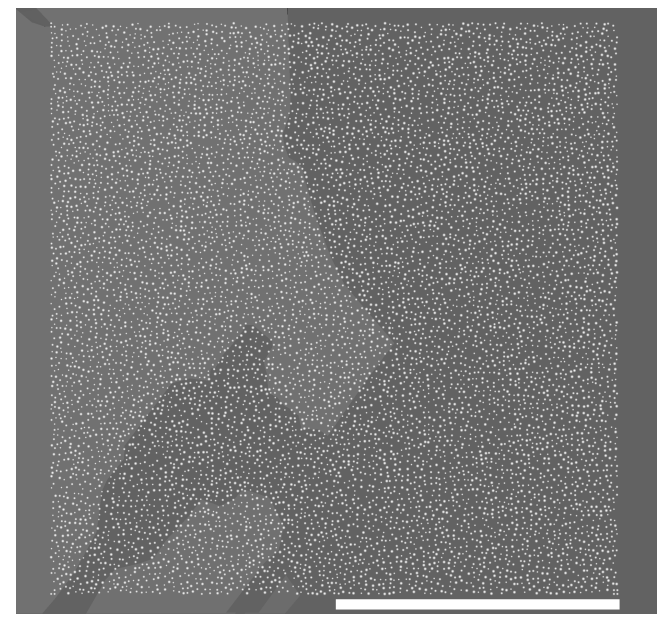

(b)

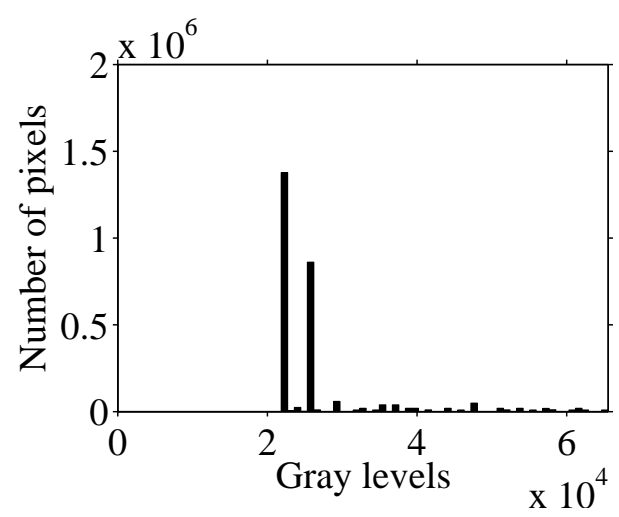

(d)

Figure 4. Reference image of the speckle built from SE image (a), and from BSE image (b). The scale bar is $200 \mu \mathrm{m}$. Gray levels histogram of the reference SE image (c), and reference BSE image (d)

DIC technique leads to a displacement uncertainty due to acquisition noise as a root mean square (RMS) value of 0.021 pixel (or $5.0 \mathrm{~nm}$ ). The latter was estimated by correlating two SE pictures with no motion applied between the two acquisitions. The RMS level of the correlation residual $\rho_{c}$ at convergence is equal to $4.3 \%$ of the dynamic range of $f$.

In Figures 6(a) and 6(b), the measured displacement respectively along the horizontal and vertical directions between the SE image and the corresponding reference are presented. At the end of the DIC calculation, gray level residuals $\rho_{c}$, defined as the difference between the corrected deformed image $\tilde{g}$ and the reference picture $f$, indicate the quality of the registration (Figure 6(c)). These residuals, from their definition, include all sources of mismatch between images that are not captured by the chosen kinematic basis (e.g., acquisition noise). Almost identical results are obtained using the BSE image, so that only the SE image will be considered thereafter.

Analytical expressions offer a suitable and convenient description of optical distortions $[3,4,5,8,9]$, which have been proposed to describe experimentally observed 


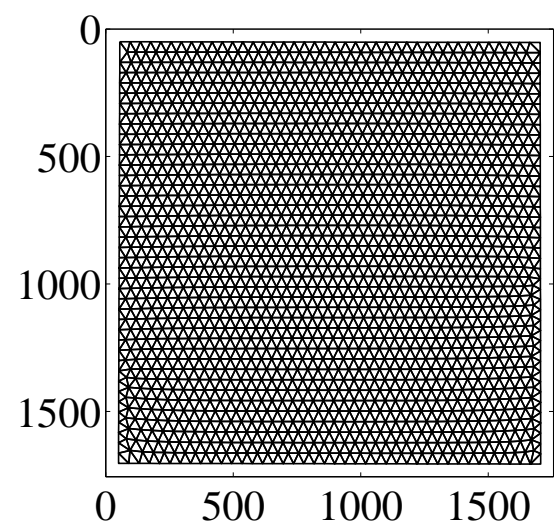

Figure 5. Unstructured mesh made of 3-noded triangular elements used for DIC calculations

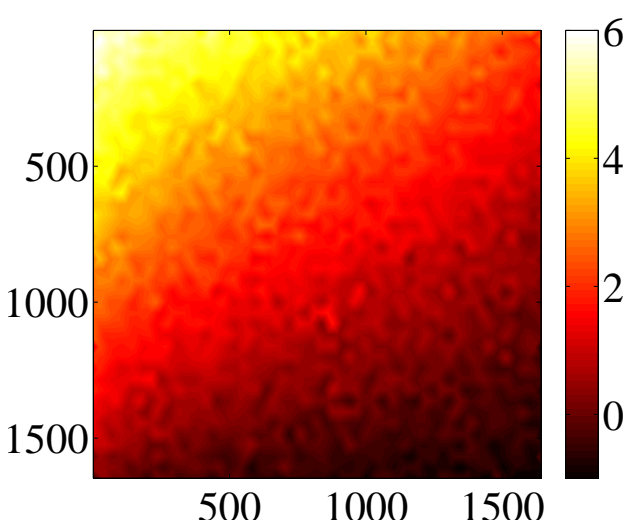

(a)

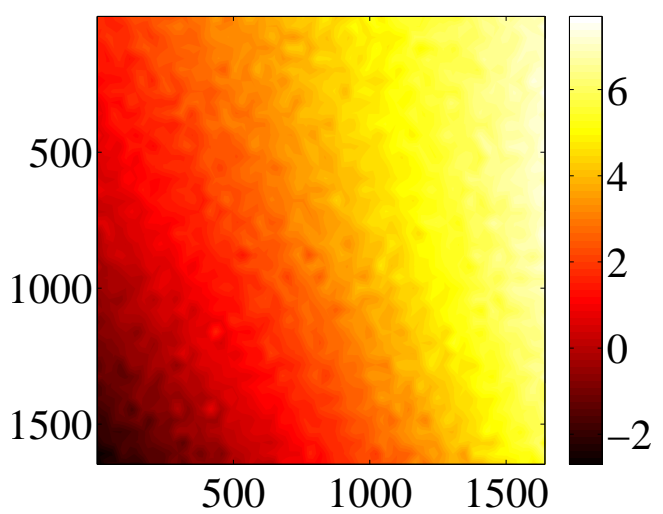

(b)

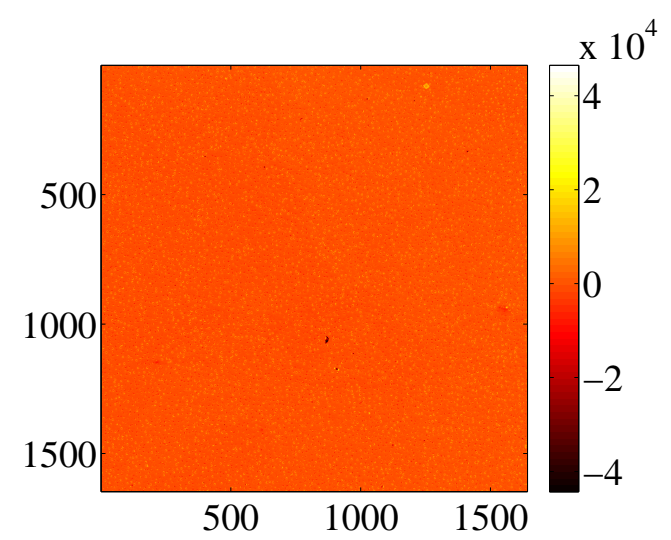

(c)

Figure 6. Displacement field expressed in pixels measured along the horizontal (a) and the vertical (b) directions between the reference image and the SE image of the speckle pattern. The physical size of one pixel is $240 \mathrm{~nm}$. Correlation residuals expressed in gray levels (c)

distortions. In this paper, a polynomial basis in the complex variables $z$ and $\bar{z}$ of maximum 
order $p$ is considered

$$
\boldsymbol{d}_{k l}(\boldsymbol{z})=\boldsymbol{z}^{k} \overline{\boldsymbol{z}}^{l} \quad 0 \leq k, 0 \leq l, k+l \leq p
$$

where $\bar{z}$ is the conjugate of $\boldsymbol{z}$. The usual expressions for radial, prismatic and decentering distortions $[8,23,24]$, may be expressed by using this series of complex shape functions. A second order expansion $p=2$ of the series has been chosen, which appeared to be sufficient to describe optical distortions. The first element of the series 1 (with complex amplitude), and the imaginary part of $\boldsymbol{z}$, represent 2D rigid body motions (i.e., translations and rotation). The projection of the displacement field measured by DIC onto the chosen basis leads to

$$
\boldsymbol{v}(\boldsymbol{z})=a_{1} \mathbf{1}+a_{2} z+a_{3} \bar{z}+a_{4} z^{2}+a_{5} z \bar{z}+a_{6} \bar{z}^{2}
$$

where $\left(a_{1}, \ldots, a_{6}\right)$ are complex-valued unknowns. Examples of the components of these trial fields are shown in Appendix A. The measured displacement field $\boldsymbol{u}$ is approximated by $\boldsymbol{v}$ by resorting to least squares minimization

$$
\left(a_{1}, \ldots, a_{6}\right)=\operatorname{argmin}\|\boldsymbol{v}-\boldsymbol{u}\|^{2}
$$

The problem to solve (9) can then be written as a linear system, whose inversion leads to the coefficients given in Table 1. The approximated field $v$ is shown in Figures 7(a) and 7(b), and the residual displacement field $\boldsymbol{\rho}=\boldsymbol{v}-\boldsymbol{u}$ in Figures 7(c) and 7(d). The RMS value of the residual displacement is 0.14 pixel (or $34 \mathrm{~nm}$ ).

Table 1. Coefficients (expressed in pixels) of the projection of the displacement field measured by DIC onto the trial fields defined in Equation (8). The physical size of one pixel is $240 \mathrm{~nm}$

\begin{tabular}{cccccc}
\hline$a_{1}$ & $a_{2}$ & $a_{3}$ & $a_{4}$ & $a_{5}$ & $a_{6}$ \\
\hline $3.3+1.5 i$ & $-2.7-5.7 i$ & $0.23+1.3 i$ & $-0.089-0.82 i$ & $-0.78+0.74 i$ & $-1.3+0.61 i$ \\
\hline
\end{tabular}

The residual displacement fields still contains some kinematic signal, since the RMS value $(0.14$ pixel or $34 \mathrm{~nm})$ is about seven times the standard displacement resolution $(0.021$ pixel or $5.0 \mathrm{~nm})$. By comparison, the projection of the displacement field measured by DIC on spline functions, using third-order Bézier curves with 16 control points $(4 \times 4$ grid), leads to an RMS value of the residual displacement of 0.09 pixel (or $22 \mathrm{~nm}$ ).

\subsection{SEM distortion measurements}

Several SEM images of the pattern are now used to quantify both patterning and imaging distortions. The imaging distortions classically encompass drift in addition to spatial distortions. However, the drift component can be neglected for the short sequence of about 30 minutes necessary to acquire the images [3, 4, 9], and because a field emission gun SEM was used, known to induce very limited drift when compared to conventional tungsten filaments $[3,4,9]$. 


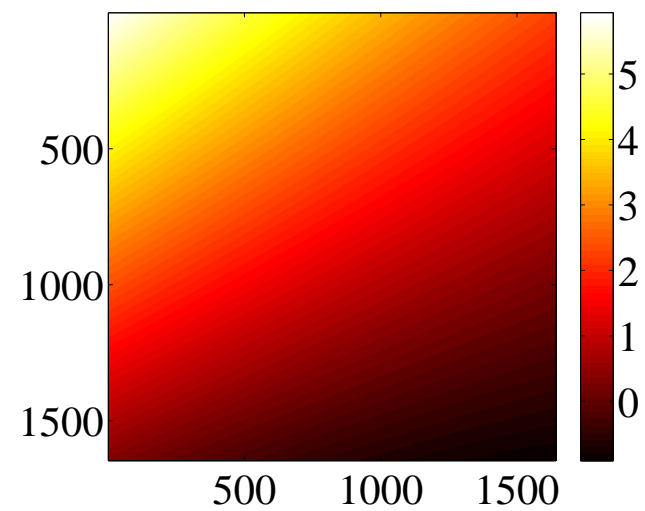

(a)

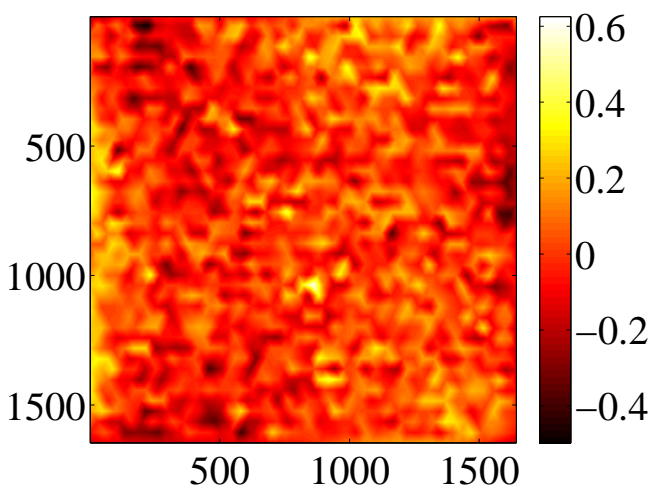

(c)

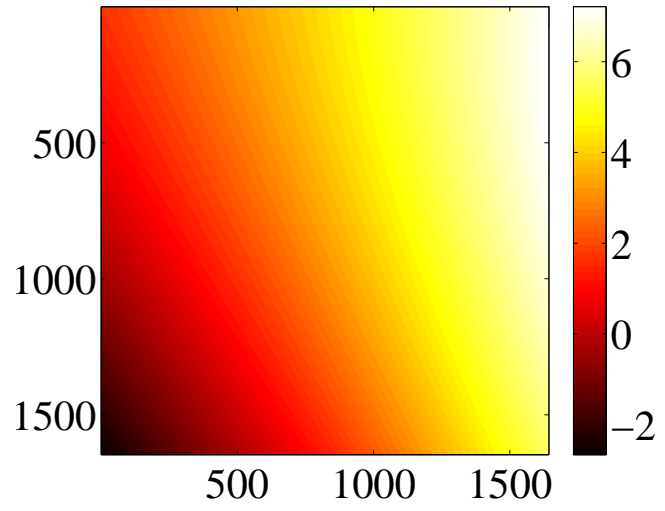

(b)

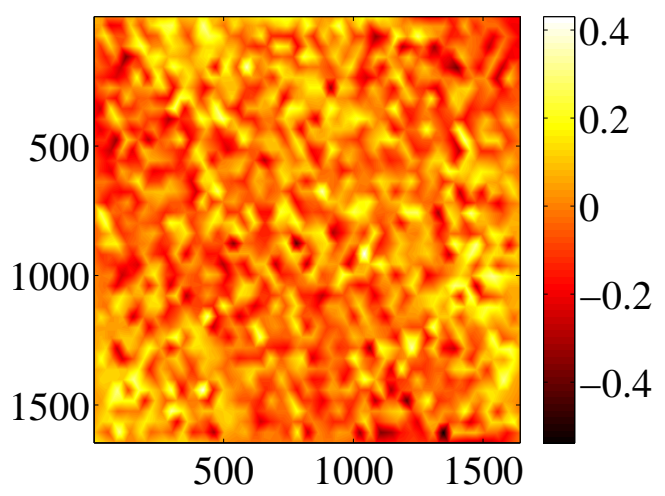

(d)

Figure 7. Projection of the displacements measured by DIC onto the basis defined in Equation (8) along the horizontal (a) and the vertical (b) directions. Difference between these projected fields and the measured fields along the horizontal (c) and the vertical (d) directions. The fields are expressed in pixels, whose physical size is $240 \mathrm{~nm}$

3.2.1. Apparent DIC motion from actual sample rotation Several evaluations of SEM distortion are sought by measuring displacements between the ideal speckle and a set of SEM images. Between each image, an increment of $10^{\circ}$ rotation of the SEM stage is applied, starting from $0^{\circ}$ up to $90^{\circ}$, keeping the imaging conditions strictly identical.

Therefore, the assumption of small displacements is no longer true for the Taylor expansion of $g(\boldsymbol{Z}(\boldsymbol{z}))$ in the DIC formulation summarized before. One may then consider finite transformation kinematics in DIC. The deformation gradient tensor $\boldsymbol{F}$ is defined as [25]

$$
\boldsymbol{F}=\nabla_{z} Z=I+\nabla_{z} u(z)
$$

where $\boldsymbol{I}$ is the identity tensor.

The same DIC strategy as previously is followed by correcting the deformed image and bringing it back onto the reference one. The transformation $\boldsymbol{Z}(\boldsymbol{z})$ is sought, and because of the intrinsic nonlinearities of the problem, an iterative approach is chosen. At step $n$ of the algorithm, an approximation of the transformation is denoted by

$$
Z \approx \chi^{(n)}(z)
$$


and the corresponding displacement reads

$$
\boldsymbol{v}^{(n)}(\boldsymbol{z})=\chi^{(n)}(\boldsymbol{z})-\boldsymbol{z}
$$

The incremental displacement correction $\delta \boldsymbol{v}^{(n+1)}(\boldsymbol{z})$ is obtained from its definition

$$
Z=\chi^{(n)}(z)+\delta \boldsymbol{v}^{(n+1)}(z)
$$

and a Taylor expansion of the corrected-deformed image, where $\tilde{g}^{(n)}(\boldsymbol{z})$ denotes $g\left(\chi^{(n)}(\boldsymbol{z})\right)$, becomes

$$
\begin{aligned}
g(\boldsymbol{Z}(\boldsymbol{z})) & =g\left(\boldsymbol{\chi}^{(n)}(\boldsymbol{z})+\boldsymbol{\delta} \boldsymbol{v}^{(n+1)}(\boldsymbol{z})\right) \\
& =g\left(\boldsymbol{\chi}^{(n)}(\boldsymbol{z})\right)+\nabla_{\boldsymbol{Z}} g\left(\boldsymbol{\chi}^{(n)}(\boldsymbol{z})\right) \cdot \boldsymbol{\delta} \boldsymbol{v}^{(n+1)}(\boldsymbol{z}) \\
& =g\left(\boldsymbol{\chi}^{(n)}(\boldsymbol{z})\right)+\nabla_{\boldsymbol{z}} g\left(\boldsymbol{\chi}^{(n)}(\boldsymbol{z})\right) \cdot \boldsymbol{F}^{(n+1)^{-1}} \cdot \boldsymbol{\delta} \boldsymbol{v}^{(n+1)}(\boldsymbol{z}) \\
& \approx \tilde{g}^{(n)}(\boldsymbol{z})+\nabla_{\boldsymbol{z}} f(\boldsymbol{z}) \cdot \boldsymbol{F}^{(n+1)^{-1}} \cdot \boldsymbol{\delta} \boldsymbol{v}^{(n+1)}(\boldsymbol{z})
\end{aligned}
$$

It is observed that if $\boldsymbol{\delta} \boldsymbol{w}^{(n+1)}(\boldsymbol{z}) \equiv \boldsymbol{F}^{(n+1)^{-1}} \cdot \boldsymbol{\delta} \boldsymbol{v}^{(n+1)}(\boldsymbol{z})$ is introduced, then

$$
g(\boldsymbol{Z}(\boldsymbol{z}))-f(\boldsymbol{z}) \approx \tilde{g}^{(n)}(\boldsymbol{z})-f(\boldsymbol{z})+\nabla_{\boldsymbol{z}} f(\boldsymbol{z}) \cdot \boldsymbol{\delta} \boldsymbol{w}^{(n+1)}(\boldsymbol{z})
$$

hence, the standard DIC procedure (3) can be used (i.e., same matrix and second member construction) to evaluate $\delta \boldsymbol{w}^{(n+1)}$, but the incremental displacement should be corrected to

$$
\boldsymbol{\delta} \boldsymbol{v}^{(n+1)}(\boldsymbol{z})=\boldsymbol{F}^{(n+1)} \cdot \boldsymbol{\delta} \boldsymbol{w}^{(n+1)}(\boldsymbol{z})
$$

After the correction step, the updated fields read

$$
\begin{array}{ll}
\boldsymbol{F}^{(n+1)} & =\boldsymbol{F}^{(n)}+\nabla_{\boldsymbol{z}} \boldsymbol{\delta} \boldsymbol{v}^{(n+1)}(\boldsymbol{z}) \\
\boldsymbol{v}^{(n+1)}(\boldsymbol{z}) & =\boldsymbol{v}^{(n)}(\boldsymbol{z})+\boldsymbol{\delta} \boldsymbol{v}^{(n+1)}(\boldsymbol{z}) \\
\chi^{(n+1)}(\boldsymbol{z}) & =\chi^{(n)}(\boldsymbol{z})+\boldsymbol{\delta} \boldsymbol{v}^{(n+1)}(\boldsymbol{z}) \\
\tilde{g}^{(n+1)}(\boldsymbol{z}) & =g\left(\chi^{(n+1)}(\boldsymbol{z})\right)
\end{array}
$$

In the particular case of large rigid body motions and small strains, $\boldsymbol{F}^{(n)}$ is essentially a rotation matrix, $\boldsymbol{R}^{(n)}$. The rotation is evaluated at each step $n$ of the algorithm using the polar decomposition of the deformation gradient tensor

$$
\boldsymbol{F}^{(n)}=\boldsymbol{R}^{(n)} \cdot \boldsymbol{V}^{(n)}
$$

where $\boldsymbol{V}^{(n)}$ is the right stretch tensor. From the right Cauchy-Green strain tensor

$$
\boldsymbol{C}^{(n)}=\boldsymbol{F}^{(n)^{T}} \cdot \boldsymbol{F}^{(n)}
$$

it is possible to evaluate the rotation tensor

$$
\boldsymbol{R}^{(n)}=\boldsymbol{F}^{(n)} \cdot \boldsymbol{C}^{(n)^{-1 / 2}}
$$

The measured displacement fields are then projected onto the chosen basis of fields (8). The RMS value of the residual displacement is shown in Figure 8 as a function of the prescribed rotation angle of the SEM stage. The residual displacements differ very slightly for all possible rotation angles. Its mean value over this set of ten evaluations is 0.22 pixel (or $54 \mathrm{~nm}$ ) for a standard deviation of 0.009 pixel (or $2.0 \mathrm{~nm}$ ).

For each rotation angle, the residual displacement fields are similar to those obtained without rotation (see Figures 7(c) and 7(d)), but with different dynamic ranges. The average residual displacement fields are shown in Figures 9(a) and 9(b). 


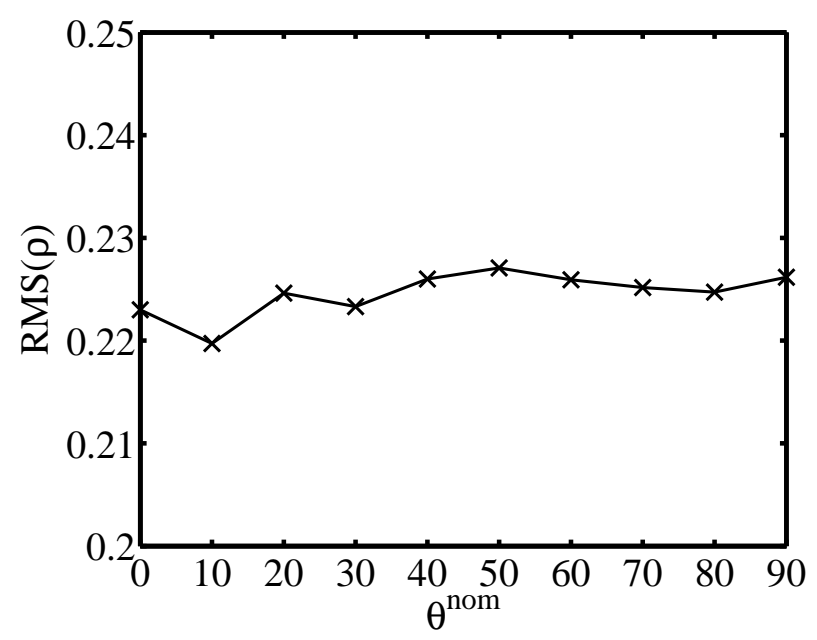

Figure 8. Change of the RMS displacement error $\rho$ in pixels as a function of the nominal rotation angle of the SEM stage (in degrees). The physical size of one pixel is $240 \mathrm{~nm}$

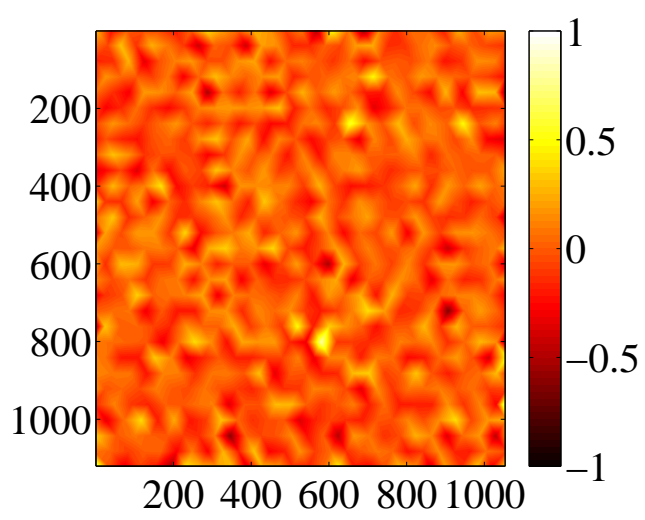

(a)

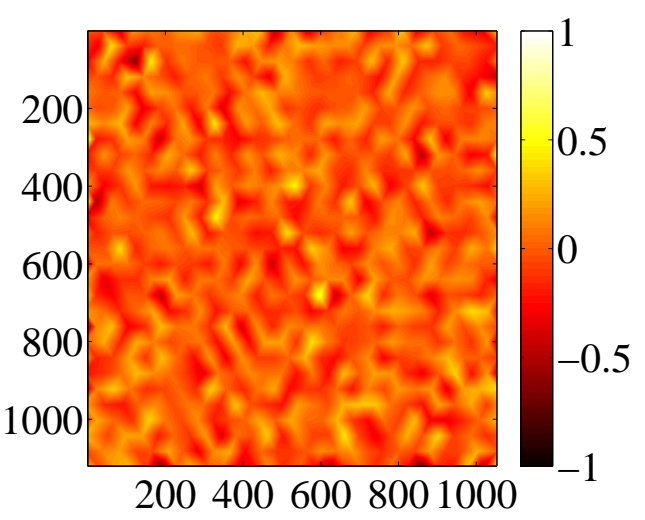

(b)

Figure 9. Average residual displacement fields along the horizontal (a) and the vertical (b) directions. The fields are expressed in pixels, whose physical size is $240 \mathrm{~nm}$

The evaluation of the rotation matrix $\boldsymbol{R}^{(n)}$ during the DIC calculation gives us a direct access to the true value of the rotation angle. Therefore, over the 9 successive rotations of $10^{\circ}$, an average of measured angle increment of $9.8^{\circ}$ is obtained, with an RMS value of $0.3^{\circ}$. This observation may result from an underestimation of the setpoint angle, or from a slight tilt of the rotation stage [24].

3.2.2. Apparent DIC motion from scan beam rotation In this part, we apply the same procedure as previously, but instead of rotating the sample using the SEM stage holder, a series of $10^{\circ}$ rotation increments of the SEM scan beam is conducted. The working conditions of the SEM are kept identical. Displacement measurements between the reference speckle and the SEM images are achieved using the same DIC method and the same mesh as discussed above. 
Similar residual displacement errors as functions of the nominal rotation angle are obtained (Figure 10), once the measured displacement fields are approximated with the chosen basis of distortion fields. The mean value of the RMS levels of residual displacements over this set of ten evaluations is 0.22 pixel (or $54 \mathrm{~nm}$ ) for a standard deviation of 0.008 pixel (or $2.0 \mathrm{~nm}$ ), which are very close to the previous levels.

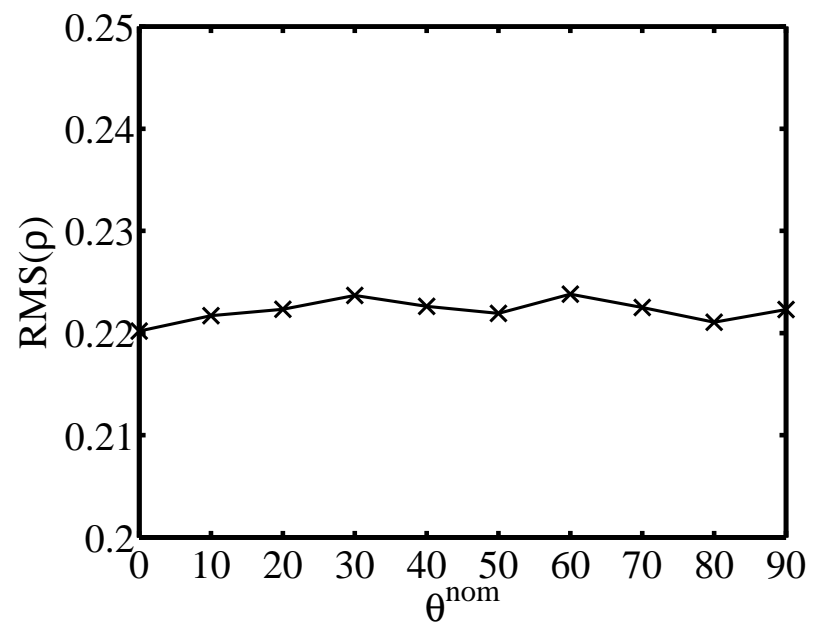

Figure 10. Change of the RMS of displacement error $\rho$ in pixels as a function of the nominal rotation angle of the scan (in degrees). The physical size of one pixel is $240 \mathrm{~nm}$

An average of measured angle increment of $10.0^{\circ}$ is now obtained, with an RMS value of $0.008^{\circ}$. With regard to this result, the scan beam rotation appears to be much more accurate than the stage rotation. It does not involve any out-of-plane displacement due to misalignments as the stage is motionless.

\section{Partition of patterning and imaging errors}

Let us now separate the relative contributions of patterning and imaging in the overall error that has been measured. Ten DIC measurements of displacement fields between SEM images rotated at ten different angles (thanks to a stage rotation every $10^{\circ}$ increment from $0^{\circ}$ to $90^{\circ}$ ) and the reference pattern are used. The ROI and the mesh are identical for each measurement.

For each rotation angle, $\theta_{k}$, the displacement measured by DIC, noted $\boldsymbol{u}^{k}$, is sought as the composition of different transformations; first a patterning distortion, then a rotation roughly known, and finally an imaging error described in Figure 11. The searched patterning error $\boldsymbol{v}_{g}$ is strictly identical for the ten displacement fields, which is to be expected since the pattern remains unchanged. Concerning the imaging distortions, $\boldsymbol{v}_{d}$, a similar assumption of stationarity is made, ignoring their time evolution.

The principle to separate these two contributions consists of expressing the apparent displacement field as a static field (the imaging distortion), and one that rotates together with the sample. Because large rotations are considered, the problem is nonlinear in the entire set of parameters needed to characterize each displacement field. However, considering one 


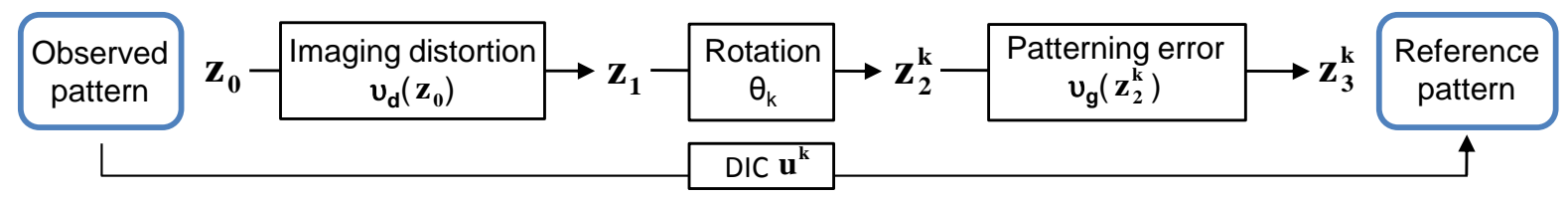

Figure 11. Schematic of the decomposition of each of the $k$ displacement measurements into imaging distortions, rotation and patterning errors

of the displacement fields as known, it is rather straightforward to determine the second and vice-versa. This procedure converges to a well defined solution in few iterations (i.e., typically of the order of 10).

The remainder of this section gives details on the formulation and solution of the problem using a similar approach as that of the previous section. The deformed coordinate system in the reference pattern image, denoted $z_{3}^{k}$, is expressed as

$$
\boldsymbol{z}_{3}^{k}=\boldsymbol{z}_{0}+\boldsymbol{u}^{k}\left(\boldsymbol{z}_{0}\right)
$$

where $\boldsymbol{u}^{k}$ is the $k$ measured displacement field.

An approximation of $\boldsymbol{z}_{3}^{k}$, denoted $\hat{\boldsymbol{z}}_{3}^{k}$ is proposed

$$
\hat{\boldsymbol{z}}_{3}^{k}=\left(\boldsymbol{z}_{0}+\boldsymbol{v}_{d}\left(\boldsymbol{z}_{0}\right)\right) e^{i\left(\theta^{k}+d \theta^{k}\right)}+\boldsymbol{v}_{g}\left(\hat{\boldsymbol{z}}_{2}^{k}\right)
$$

where $\hat{z}_{2}^{k}$ is an estimation of an intermediate coordinate system after imaging distortions and the rotation

$$
\left.\hat{\boldsymbol{z}}_{2}^{k}=\left(\boldsymbol{z}_{0}+\boldsymbol{v}_{d}\left(\boldsymbol{z}_{0}\right)\right) e^{i\left(\theta^{k}+d \theta^{k}\right.}\right)
$$

and $d \theta^{k}$ a set of unknowns introduced to allow for small fluctuations from the nominal value of the angle $\theta^{k}$ previously measured (see Section 3.2.1). The displacement fields $\boldsymbol{v}_{d}$ and $\boldsymbol{v}_{g}$ are both searched in the form of the previously described expressions of distortions (7). A second order expansion is chosen as previously (see Equation (8)), leading to two sets of complex parameters $\left(a_{1}^{k}, \ldots, a_{6}\right)$ and $\left(b_{1}, \ldots, b_{6}\right)$ to describe respectively $\boldsymbol{v}_{d}$ and $\boldsymbol{v}_{g}$. Because they control the $2 \mathrm{D}$ rigid body motions, the parameters $a_{1}^{k}$ are specific to each field $k$.

The rotation offsets $d \theta^{k}$ are very small and, consistently with the small correction assumption, second order cross-products of unknowns are neglected so that Equation (22) becomes

$$
\hat{\boldsymbol{z}}_{3}^{k}=\left(\boldsymbol{z}_{0}+\boldsymbol{v}_{d}\left(\boldsymbol{z}_{0}\right)+i d \theta^{k} \boldsymbol{z}_{0}\right) e^{i \theta^{k}}+\boldsymbol{v}_{g}\left(\hat{\boldsymbol{z}}_{2}^{k}\right)
$$

An additional constraint prescribes the imaginary part of $a_{2}$ to zero since the rotation is described by $\theta^{k}$ and $d \theta^{k}$.

The quality of the proposed deconvolution is estimated by the error fields

$$
\boldsymbol{r}^{k}=\boldsymbol{z}_{3}^{k}-\hat{z}_{3}^{k}
$$

The choice was made to estimate $\boldsymbol{v}_{d}$ and $\boldsymbol{v}_{g}$ one after the other and not simultaneously. Thus two linear systems are solved instead of one nonlinear system by resorting to least squares fit. However, approximation errors are cumulated and should be reduced. A loop is set 
up to first update $\boldsymbol{v}_{d}^{(n+1)}$ from $\boldsymbol{v}_{g}^{(n)}$, then $\boldsymbol{v}_{g}^{(n+1)}$ and iteratively continue, until the convergence of the error fields $\boldsymbol{r}^{k}$ is reached. As initialization, the patterning errors $\boldsymbol{v}_{g}^{(0)}$ are neglected.

In Figure 12, imaging distortions and patterning errors obtained at convergence are shown. The change of the residual fields $\boldsymbol{r}^{k}$ between the first iteration and the final iteration is presented in Figure 13 as functions of the rotation angle of the pattern. Both horizontal and vertical components of the ten error fields are shown in Figure 14. A low frequency kinematic signal still appears in some of the error fields. It was checked that richer bases (i.e., higher order terms of the series expansion (7)) for $\boldsymbol{v}_{d}$ or $\boldsymbol{v}_{g}$ do not allow for their reduction.

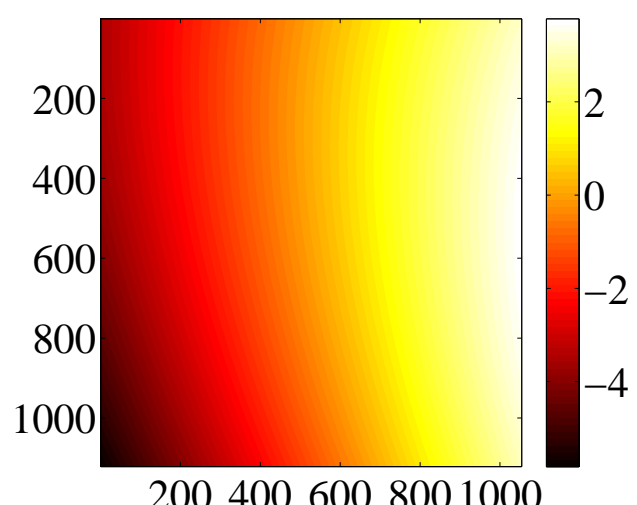

(a)

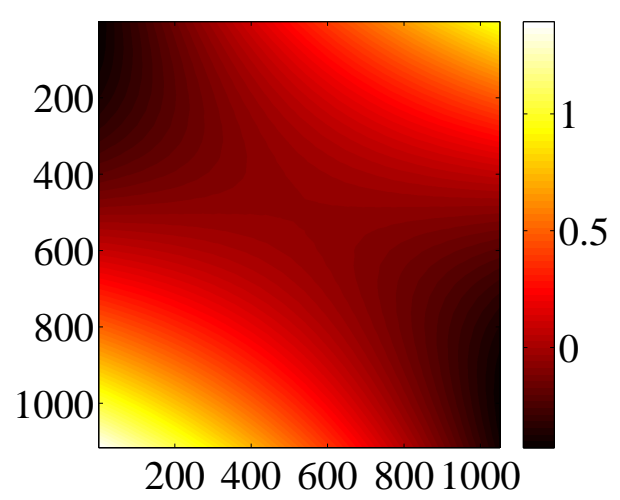

(c)

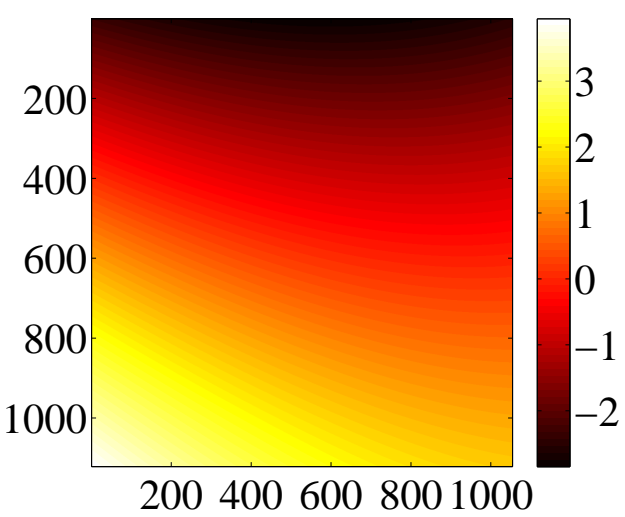

(b)

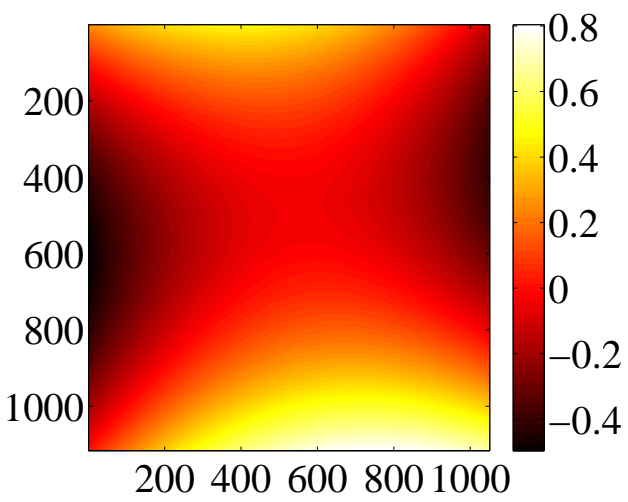

(d)

Figure 12. Imaging distortions along the horizontal (a) and the vertical (b) directions, and patterning errors along the horizontal (c) and the vertical (d) directions at convergence of the deconvolution. The fields are expressed in pixels, whose physical size is $240 \mathrm{~nm}$

\section{Conclusion and perspectives}

Surface marking of steel samples by e-beam microlithography with known reference (i.e., numerically-generated random pattern) has been characterized by means of digital image correlation (DIC). As picture acquisitions are obtained with SEM instruments, the measured displacement fields from the registration of the reference and imaged patterns is a combination of patterning and imaging biases. Several evaluations of the distortions have been performed, 


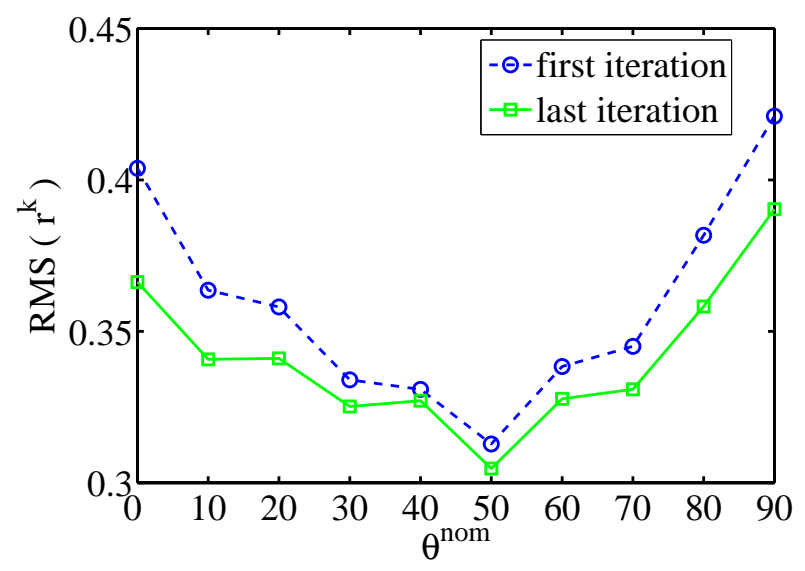

Figure 13. Change of the RMS value of the residual fields in pixels as a function of the nominal rotation angle of the pattern (in degrees). The physical size of one pixel is $240 \mathrm{~nm}$

after a series of rotations of the observed pattern and of the SEM scan beam. A second order polynomial basis revealed sufficient to capture most of the distortions, which turned out to be very comparable in SE and BSE modes and for the different rotation angles. To separate the global errors into two fields, one for patterning and one for imaging, it is proposed to analyze a series of images acquired after rotation of the specimen by different angles. An iterative scheme based on the linearization of the displacement errors allows for convergence in few iterations. The patterning is shown to involve much less errors (in the $400 \mathrm{~nm}$ range) than the imaging distortions (in the $2 \mu \mathrm{m}$ range).

Since very large rotations are prescribed (i.e., up to $90^{\circ}$ ), finite transformation kinematics has been implemented in the DIC formulation. The basic structure of the code remains unchanged. The only modification is related to the displacement corrections that are evaluated at each iteration. They require an estimate of the mean component of the deformation gradient tensor.

Once the proposed calibration has been performed, the random part of the measurement error and some additional drifts can be evaluated by acquiring different images of the same sample without any motion of the SEM stage. When these last steps are carried out, the SEM user has a better evaluation of the measurement errors when registering images via DIC. Despite the difficulties and restrictions associated with sample patterning at small scales, and the use of imaging devices not initially tailored for kinematic measurements, the errors obtained on displacement fields are very small. Some additional errors will arise when the sample deforms, for example because the local texture imaged by the SEM can evolve upon straining due to slip band accumulation and topographic variations. This will have to be addressed in future studies since it is desirable to characterize and improve the kinematic measurements conducted at microstructural scales for both low and high strain values.

The SEM in-situ material testing combined with DIC will then be highly beneficial for the study of plasticity and its onset, and for the analysis of damage involving larger strains. With such data, parameters of crystal plasticity laws can be determined by coupling full field 


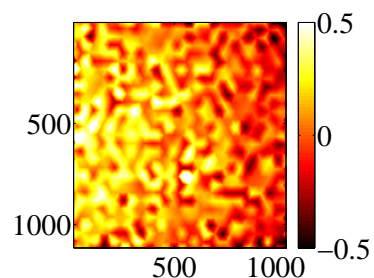

(a) $r_{x}^{1}$

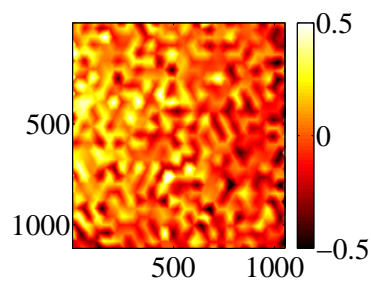

(e) $r_{x}^{3}$

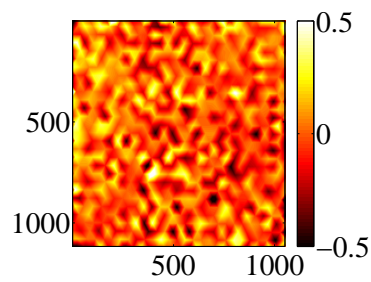

(i) $r_{x}^{5}$

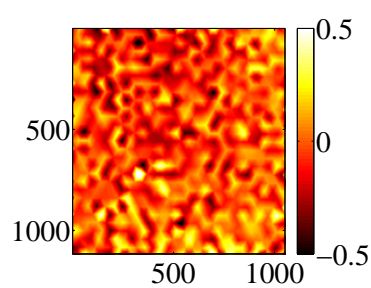

(m) $r_{x}^{7}$

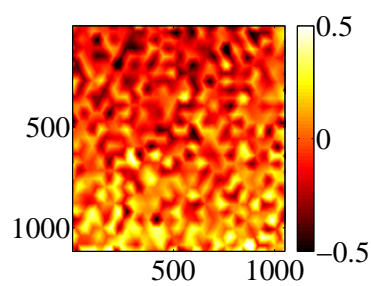

(q) $r_{x}^{9}$

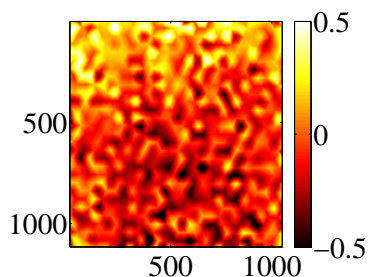

(b) $r_{y}^{1}$

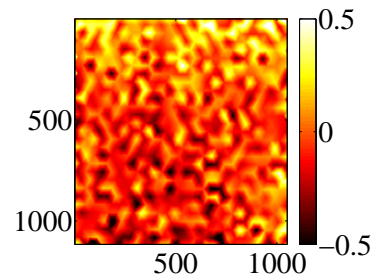

(f) $r_{y}^{3}$

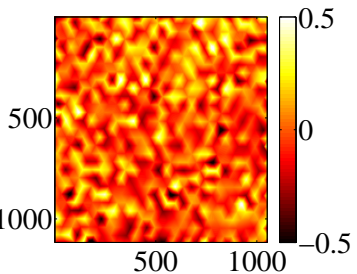

(j) $r_{y}^{5}$

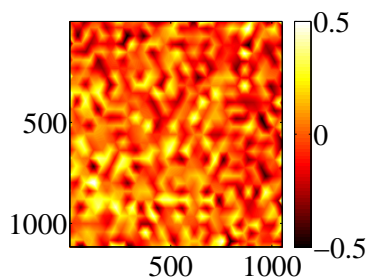

(n) $r_{y}^{7}$

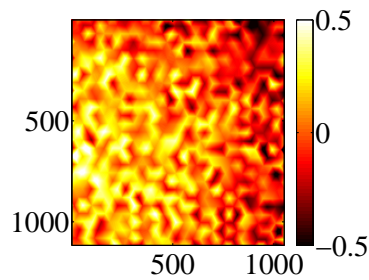

(r) $r_{y}^{9}$

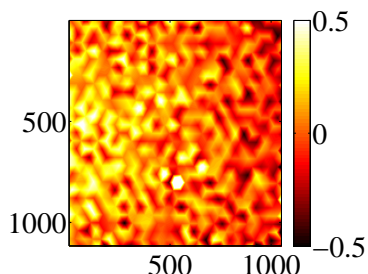

(c) $r_{x}^{2}$

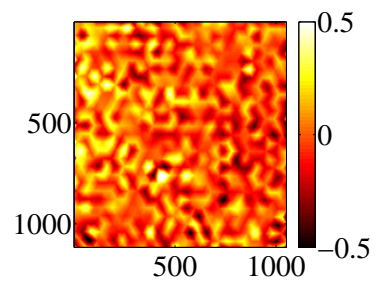

(g) $r_{x}^{4}$

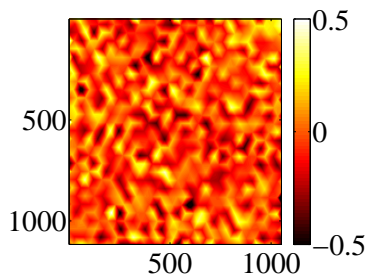

(k) $r_{x}^{6}$

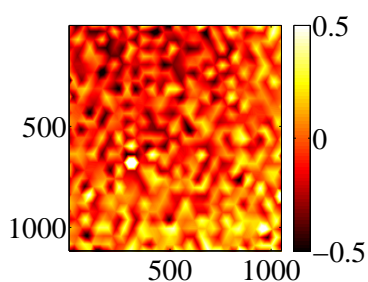

(o) $r_{x}^{8}$

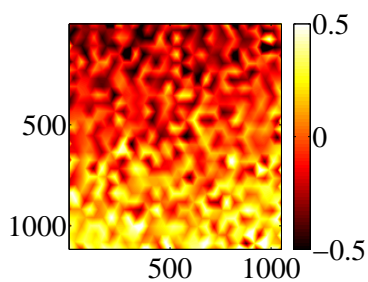

(s) $r_{x}^{10}$

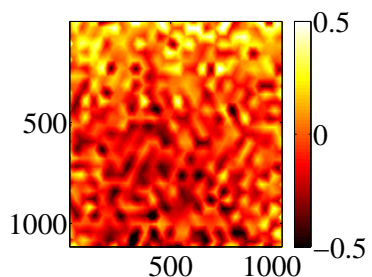

(d) $r_{y}^{2}$

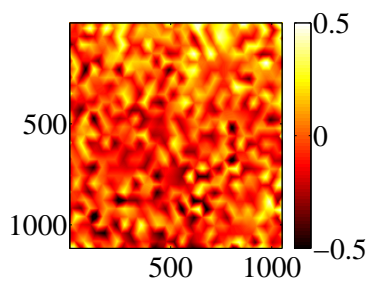

(h) $r_{y}^{4}$

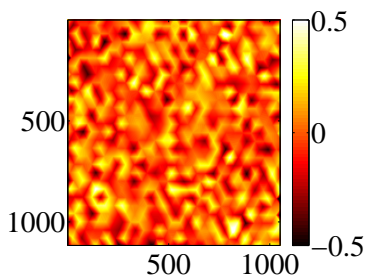

(1) $r_{y}^{6}$

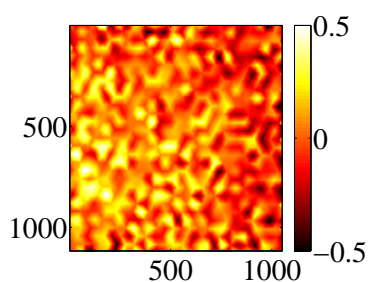

(p) $r_{y}^{8}$

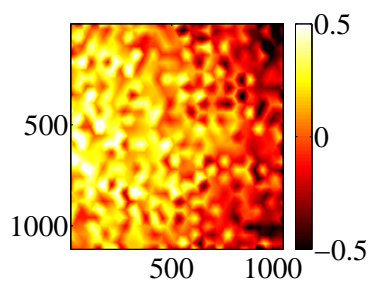

(t) $r_{y}^{10}$

Figure 14. Ten error fields in pixels of the partition at convergence. $r_{x}^{k}$ is the component of $r^{k}$ along the horizontal direction, and $r_{y}^{k}$ along the vertical direction. The physical size of one pixel is $240 \mathrm{~nm}$

measurements and finite element simulations. 


\section{Acknowledgments}

The authors acknowledge the financial support of EDF within R\&D LOCO and PERFORM60 (www.perform60.net) projects. Michel Mahé, Nicolas Brynaert and the Electronic Microscopy Laboratory at Les Renardières are thanked for their help in preparing and conducting the SEM acquisitions. Finally, Nicolas Rupin, François Curtit and Sylvain Leclercq are thanked for the fruitfull discussions. 
Appendix A. Displacement fields of the second order expansion of the basis expressed in Equation (7)

The measured displacement fields are approximated as linear combinations of the following fields with complex-valued amplitudes. Thus, a full display of the basis also includes the same five fields but with a swap of the columns and with an opposite sign of one column.

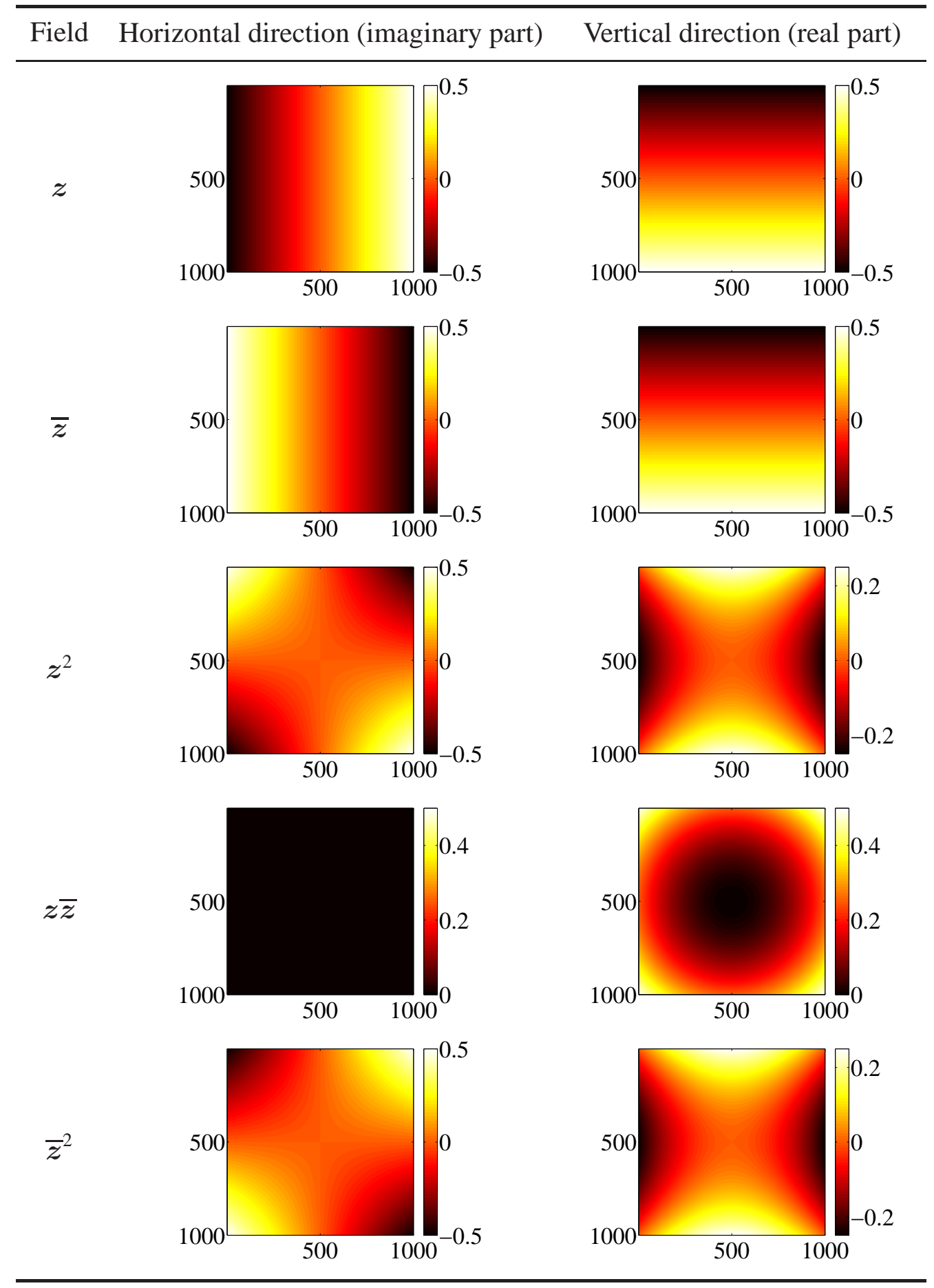




\section{Reference}

[1] F. Roters, P. Eisenlohr, L. Hantcherli, D.D. Tjahjanto, T.R. Bieler, and D. Raabe. Overview of constitutive laws, kinematics, homogenization and multiscale methods in crystal plasticity finite-element modeling: Theory, experiments, applications. Acta Materialia, 58(4):1152-1211, 2010.

[2] F. Latourte, N. Rupin, M. He, A. Parrot, and S. Leclercq. Full field measurements used for assessing industrial issues - two examples. Procedia IUTAM, 4(0):92-105, 2012.

[3] M. A. Sutton, N. Li, D. C. Joy, A. P. Reynolds, and X. Li. Scanning electron microscopy for quantitative small and large deformation measurements part i: Sem imaging at magnifications from 200 to 10.000. Experimental Mechanics, 47:775-787, 2007.

[4] M. A. Sutton, N. Li, D. Garcia, N. Cornille, J.-J. Orteu, S. R. McNeill, H. W. Schreier, X. Li, and A. P. Reynolds. Scanning electron microscopy for quantitative small and large deformation measurements part ii: Experimental validation for magnifications from 200 to 10.000. Experimental Mechanics, 47:789-804, 2007.

[5] T. Zhu, M. A. Sutton, N. Li, J.-J. Orteu, N. Cornille, X. Li, and A. P. Reynolds. Quantitative stereovision in a scanning electron microscope. Experimental Mechanics, 51:97-109, 2011.

[6] C. Li, Z. Liu, and H. Xie. A measurement method for micro 3d shape based on grids-processing and stereovision technology. Measurement Science and Technology, 24:9pp, 2013.

[7] E. Héripré, M. Dexet, J. Crépin, L. Gélébart, A. Roos, M. Bornert, and D. Caldemaison. Coupling between experimental measurements and polycrystal finite element calculations for micromechanical study of metallic materials. International Journal of Plasticity, 23(9):1512-1539, 2007.

[8] D.C. Brown. Decentering distortion of lenses. Photogrammetric Engineering, 32(3):444-462, 1966.

[9] M. A. Sutton, N. Li, D. Garcia, N. Cornill, J. J. Orteu, S. R. McNeill, H. W. Schreier, and X. Li. Metrology in a scanning electron microscope: theoretical developments and experimental validation. Measurement Science and Technology, 17:2613-2622, 2006.

[10] C. Ru, Y. Zhang, Y. Sun, Y. Zhong, X. Sun, D. Hoyle, and I. Cotton. Automated four-point probe measurement of nanowires inside a scanning electron microscope. IEEE Transactions on Nanotechnology, 10:674-681, 2011.

[11] A. Allais, M. Bornert, T. Bretheau, and D. Caldemaison. Experimental characterization of the local strain field in an heterogeneous elastoplastic material. Acta Metalurgica Materialia, 42(11):3865-3880, 1994.

[12] P. Doumalin, M. Bornert, and J. Crépin. Caractérisation de la répartition de la déformation dans les matériaux hétérogènes. Mécanique $\mathcal{E}$ Industries, 4(6):607-617, 2003.

[13] P. Doumalin. Microextensométrie locale par corrélation d'images numériques : Application aux études micromécaniques par microscopie électronique à balayage. $\mathrm{PhD}$ thesis, Ecole Polytechnique, 2000.

[14] A. D. Kammers and S. Daly. Small-scale patterning methods for digital image correlation under scanning electron microscopy. Measurement Science and Technology, 22(12):12pp, 2011.

[15] S. A. Collette, M. A. Sutton, P. Miney, A. P. Reynolds, Xiaodong Li, P. E. Colavita, W. A. Scrivens, Y. Luo, T. Sudarshan, P. Muzykov, and M. L. Myrick. Development of patterns for nanoscale strain measurements: I. fabrication of imprinted au webs for polymeric materials. Nanotechnology, 15(12):1812-1817, 2004.

[16] N. Li, M. A. Sutton, X. Li, and H. W. Schreier. Full-field thermal deformation measurements in a scanning electron microscope by 2d digital image correlation. Experimental Mechanics, 48:635-646, 2008.

[17] W. Scrivens, Y. Luo, M. A. Sutton, S. Collette, M. Myrick, P. Miney, P. Colavita, A. P. Reynolds, and X. Li. Development of patterns for digital image correlation measurements at reduced length scales. Experimental Mechanics, 47:63-77, 2007.

[18] T.A. Berfield, J.K. Patel, R.G. Shimmin, P.V. Braun, J. Lambros, and N.R. Sottos. Micro- and nanoscale deformation measurement of surface and internal planes via digital image correlation. Experimental Mechanics, 47:51-62, 2007.

[19] J.-L. Regolini. Technologie de fabrication de la microélectronique. opérations élémentaires. Techniques de l'ingénieur, E2410, 1991.

[20] H. Leclerc, J.-N. Périé, S. Roux, and F. Hild. Integrated digital image correlation for the identification of 
mechanical properties. MIRAGE 2009, LNCS, 5496:161-171, 2009.

[21] F. Hild and S. Roux. Comparison of local and global approaches to digital image correlation. Experimental Mechanics, 52:1503-1519, 2012.

[22] G. Besnard, F. Hild, and S. Roux. "finite-element" displacement fields analysis from digital images: Application to portevin-le châtelier bands. Experimental Mechanics, 46:789-803, 2006.

[23] M. A. Sutton, J.-J. Orteu, and H. Schreier. Image Correlation for Shape, Motion and Deformation Measurements: Basic Concepts,Theory and Applications. Springer handbook of experimental solid mechanics, Berlin, 2009.

[24] J.E. Dufour, F. Hild, and S. Roux. Integrated digital image correlation for the evaluation and correction of optical distortions. Optics and Lasers in Engineering, (submitted for publication), 2013.

[25] C. Truesdell and W. Noll. The non-linear field theories of mechanics. In S. Flügge, editor, Handbuch der Physik, volume III/3. Springer-Verlag, Berlin, 1965. 